\title{
Effect of dexmedetomidine hydrochloride combination with conventional anesthesia on serum cortisol, inflammatory factors and cellular immunity during surgery on children with congenital anus atresia
}

\author{
Aihua Liu, Lichuan Tian, Fang Yin* \\ Department of Anaesthesia, Cangzhou People's Hospital, Cangzhou City, China \\ *For correspondence: Email: jn1336@163.com \\ Sent for review: 4 April 2018 \\ Revised accepted: 26 June 2018
}

\begin{abstract}
Purpose: To study the effect of a combination of dexmedetomidine hydrochloride (DXM) and conventional anesthesia on surgical anesthesia in pediatric congenital anoplasty.

Methods: Fifty children with congenital anus atresia were divided into control and study group. Both groups underwent anal angioplasty or colostomy, with the control group under conventional anesthesia, while the study group received both conventional anesthesia and DXM. Heart beat rate, arterial blood pressure, changes in oxygen saturation were recorded. Blood loss were noted. Venous blood was collected at various time points for assay of serum cortisol, IL-6, and T-lymphocyte subsets.

Results: The arterial pressure and heart beat of the study group at $T_{1}$ and $T_{2}$ were significantly lower than those of the control group $(p<0.05)$. Blood loss was significantly lower in the study group than in the control group ( $p<0.05)$. Serum cortisol and IL-6 levels at $T_{1}, T_{2}$ and $T_{3}$ in both groups significantly increased, compared with $T_{0}$, with levels in the study group significantly lower than those in the control group $(p<0.05)$. Values of $C D 3+, C D 4+$ and $C D 4+/ C D 8+$ in both groups significantly decreased at $T_{2}$, when compared with corresponding values at $T_{0}$, while the levels in the study group were significantly lower than those in the control group $(p<0.05)$.

Conclusion: Dexmedetomidine hydrochloride combined with conventional anesthesia is more effective than conventional anesthesia during surgery on congenital anal atresia.
\end{abstract}

Keywords: Dexmedetomidine hydrochloride, Congenital anal atresia, Cortisol, IL-6, T-lymphocyte subsets

\footnotetext{
This is an Open Access article that uses a funding model which does not charge readers or their institutions for access and distributed under the terms of the Creative Commons Attribution License (http://creativecommons.org/licenses/by/4.0) and the Budapest Open Access Initiative (http://www.budapestopenaccessinitiative.org/read), which permit unrestricted use, distribution, and reproduction in any medium, provided the original work is properly credited.
}

Tropical Journal of Pharmaceutical Research is indexed by Science Citation Index (SciSearch), Scopus, International Pharmaceutical Abstract, Chemical Abstracts, Embase, Index Copernicus, EBSCO, African Index Medicus, JournalSeek, Journal Citation Reports/Science Edition, Directory of Open Access Journals (DOAJ), African Journal Online, Bioline International, Open-J-Gate and Pharmacy Abstracts

\section{INTRODUCTION}

Congenital anal atresia is a disease of digestive tract dysplasia during fetal growth. The incidence of congenital anal atresia ranks top in digestive system abnormalities, and is $0.028 \%$ in China
[1]. Newborns with this disease should be treated by surgery as soon as possible after birth [2]. Due to developmental immaturity, the newborns are different from adults in several aspects such as pharmacological properties, and they are more sensitive to pain. 
In order to reduce their stress responses during perioperative period, an anesthesia plan focusing on their uniqueness should be used. Pediatric anoplasty (balloon angioplasty) is long, and with a large incision. Thus, it is necessary to keep stable hemodynamics during the surgery, weaken stress responses, and reduce its inhibitory effect on the children's immune system, so as to promote anesthetic effect and postoperative recovery [2]. Dexmedetomidine hydrochloride (DXM) [3], a new narcotic drug, is an adrenal receptor agonist with high selectivity and good sedative and analgesic effects, which has been widely used in perioperative analgesia for a variety of surgeries [4]. It can significantly cut down pain sensation during operation, lessen injury due to stress response, and decrease physical and psychological damage, thereby quickening postoperative recovery [5]. In the present study, DXM was used in the surgery for pediatric congenital anal atresia, and its effect on hemodynamics, cortisol, inflammatory factors and cellular immunity were determined.

\section{EXPERIMENTAL}

\section{Study subjects}

A total of 50 children with congenital anal atresia in pediatric surgery from July 2016 to July 2017 were enrolled in the study. There were 34 males and 16 females, weighing between 2.1 and 4.3 $\mathrm{kg}$. Anoplasty was used for 31 cases, while the others underwent colostomy. The subjects were coded based on their birth time, and randomly divided into control and study groups (25 per group).

Inclusion criteria: The inclusion criteria were: children diagnosed with congenital anal atresia; patients with ASA grade I II and children whose parents signed informed consent.

Exclusion criteria: children whose conditions were complicated with disorders of circulatory system, respiratory system, liver and kidney and children whose conditions were complicated with congenital immunological deficiency.

This research was approved by the Ethical Committee of Cangzhou People's Hospital (approval no. is 20170231 - 3) and carried out according to the Declaration of Helsinki promulgated in 1964 as amended in 1996 [6].

\section{Anesthesia methods}

Intake of milk or formula was not allowed $4 \mathrm{~h}$ before surgery, and drinking was discontinued at least $2 \mathrm{~h}$ before surgery. The children were injected with $10 \mu \mathrm{g} / \mathrm{kg}$ scopolamine $30 \mathrm{~min}$ before surgery, and those with severe abdominal distension underwent gastrointestinal decompression. In order to effect preoperative correction of dehydration, sodium-potassiummagnesium-calcium glucose injection (Jiangsu Hengrui Medicine Co. Ltd, batch number: Guoyaozhunzi H20103161) was given to the children through a vein-detained needle. The operating room temperature was set at $\geq 25^{\circ} \mathrm{C}$, and the children were given oxygen for denitrogen on entering the operating room.

Their ECG, HR, RR, $\mathrm{SpO}_{2}$, and bispectral index of the electroencephalogram (BIS) were monitored. Following anesthesia induction with 1 $3 \%$ sevoflurane (Jiangsu Hengrui Medicine $\mathrm{CO}$, Ltd, batch number: Guoyaozhunzi $\mathrm{H} 2007171$ ), $500 \mu \mathrm{g} / \mathrm{kg}$ atracurium (manufacturer: Jiangsu Hengrui Medicine CO. Ltd, batch number: Guoyaozhunzi H20060869) was given intravenously through the detained needle. Two minutes later, the children were intubated and mechanically ventilated. During the operation, anesthesia was maintained with $1 \sim 2 \%$ sevoflurane and remifentanil $(0.3 \mu \mathrm{gg} / \mathrm{kg} / \mathrm{h})$ (Jiangsu Nhwa Pharmaceutical Co. Ltd.; batch number: Guoyaozhunzi H20143315) administrated using a micro infusion pump. In order to keep muscle from relaxation, tracurium (300 $\mu \mathrm{g} / \mathrm{kg} / \mathrm{h})$ was administered by a micro infusion pump.

The children in the observation group were injected with $0.5 \mu \mathrm{g} / \mathrm{kg} / \mathrm{h}$ DXM (Jiangsu Hengrui Medicine CO. Ltd; batch number: Guoyaozhunzi H20090251) through a micro vein pump, which injection was completed in $5 \mathrm{~min}$. Patients in the control group were given normal saline by the same route. All children were observed for their BIS which was maintained between $40 \sim 50$. Drug administration was discontinued in both groups 5 min prior to the end of surgery. After operation, all children received intravenous injection of neostigmine $(20 \mu \mathrm{g} / \mathrm{kg})$ and atropine $(10 \mu \mathrm{g} / \mathrm{kg})$ to arrest muscle relaxation, and were sent to recovery room after resuscitation.

\section{Study parameters}

The heart rate $(\mathrm{HR})$, mean arterial pressure and blood oxygen saturation $\left(\mathrm{SpO}_{2}\right)$ of the two groups before anesthetic induction $\left(T_{0}\right)$, at the beginning $\left(T_{1}\right)$ and the end of surgery $\left(T_{2}\right)$, and day 1 after surgery $\left(T_{3}\right)$ were compared. Besides, venous blood was collected for determination of cortisol and IL-6 using ELISA method, and changes in peripheral T-cell subsets by flow cytometry (Beckman Coulter Inc). The operation time and blood loss during surgery in the two 
groups were recorded, and the differences in time taken for anesthesia recovery, length of stay in recovery room, and incidence of postoperative agitation, were compared.

\section{Statistical analysis}

All data were analyzed by software SPSS18.0. Measurement data are presented as mean $\pm S D$, and analyzed using $t$-test, while the enumeration data were analyzed by chi-squire $\left(\chi^{2}\right)$ test. Values of $p<0.05$ were taken as indicating statistically significant.

\section{RESULTS}

\section{Characteristics of study subjects}

There were no differences in sex, body weight, operation methods and birth time between the two groups $(p>0.05)$.

Operation time, blood loss, time taken for anesthesia recovery, and length of stay in recovery room

Although there were no significant differences in operation time, time taken for anesthesia recovery, and length of stay in recovery room between the two groups $(p>0.05)$, blood loss in the observation group was significantly lower than in the control group $(p<0.05)$.
Hemodynamic indices and $\mathrm{SpO}_{2}$ at each time during surgery

The $\mathrm{SpO}_{2}$ of the two groups was kept to $99 \pm 1$ $\%$ at each time point, with no statistical difference. However, the MVP and HR values of the control group were significantly increased at $\mathrm{T}_{1}$ and $\mathrm{T}_{2}(p<0.05)$, while MVP and HR did not change appreciably at each time point $(p>0.05)$. In addition, the MVP and HR of the study group were much lower than those of the control group $(p<0.05)$. These results are shown in Table 3.

\section{Levels of cortisol and IL-6 in the two groups at each time point}

Results in Table 4 indicate that compared to $\mathrm{T}_{0}$, cortisol and IL-6 levels of both groups increased at $T_{1}, T_{2}$ and $T_{3}$, while cortisol and IL- 6 levels were lower in the study group than corresponding levels in the control group $(p<$ 0.05).

\section{Comparison of T-cell subsets of the two groups at various time points}

Both groups had decreases in CD3 +, CD4 + and CD4 + / CD8 + at $T_{2}$ and $T_{3}$, relative to $T_{1}$, but the levels of these indexes were much higher in the study group than in the control group $(p<0.05$, Table 5).

Table 1: General characteristics of the patients (mean \pm SD)

\begin{tabular}{lccccc}
\hline Group & $\mathbf{N}$ & Sex (male/female) & Anoplasty/colostomy & Birth time (h) & Body weight (kg) \\
\hline Study & 25 & $16 / 9$ & $15 / 10$ & $25.9 \pm 5.8$ & $3.2 \pm 0.7$ \\
Control & 25 & $18 / 7$ & $16 / 9$ & $24.5 \pm 6.1$ & $3.1 \pm 0.9$ \\
$\mathrm{~T}\left(\mathrm{X}^{<}\right)$ & - & $(0.367)$ & 0.084 & 0.832 & 0.439 \\
$P$ & - & 0.544 & 0.771 & 0.409 & 0.663 \\
\hline
\end{tabular}

Table 2: Operation time, blood loss, time taken for anesthesia recovery, and length of stay in recovery room between the study and control groups (mean \pm SD)

\begin{tabular}{lccccc}
\hline Group & $\mathbf{N}$ & $\begin{array}{c}\text { Operation time } \\
(\mathbf{m i n})\end{array}$ & $\begin{array}{c}\text { Blood loss } \\
(\mathbf{m L})\end{array}$ & $\begin{array}{c}\text { Time taken for } \\
\text { anesthesia recovery } \\
(\mathbf{m i n})\end{array}$ & $\begin{array}{c}\text { Length of stay in } \\
\text { recovery room }(\mathbf{m i n})\end{array}$ \\
\hline Study & 25 & $73.4 \pm 14.6$ & $11.2 \pm 3.4$ & $36.3 \pm 6.9$ & $68.2 \pm 12.4$ \\
Control & 25 & $72.7 \pm 13.5$ & $18.2 \pm 2.9$ & $34.4 \pm 6.4$ & $64.3 \pm 16.5$ \\
$T$ & - & 0.176 & -7.832 & 1.009 & 0.945 \\
$P$ & - & 0.861 & $<0.001$ & 0.318 & 0.349 \\
\hline
\end{tabular}

Table 3: Heart rate and blood pressure of the two groups during surgery (mean \pm SD)

\begin{tabular}{lccccc}
\hline \multirow{2}{*}{ Group } & Index & T0 & T1 & T2 & T3 \\
\hline \multirow{2}{*}{ Study } & Heart rate $($ beats $/ \mathrm{min})$ & $136.1 \pm 9.7$ & $138.2 \pm 9.7 \#$ & $137.4 \pm 10.1 \#$ & $133.5 \pm 8.1$ \\
& Arterial Pressure $(\mathrm{kPa})$ & $10.1 \pm 0.8$ & $10.5 \pm 0.7 \#$ & $10.4 \pm 1.0 \#$ & $10.0 \pm 0.9$ \\
\multirow{2}{*}{ Control } & Heart rate $($ beats $/ \mathrm{min})$ & $135.5 \pm 9.0$ & $150.4 \pm 8.5^{\mathbf{4}}$ & $149.2 \pm 9.9^{\mathbf{4}}$ & $134.4 \pm 7.8$ \\
& Arterial pressure $(\mathrm{kPa})$ & $10.2 \pm 0.9$ & $13.2 \pm 0.7^{\mathbf{4}}$ & $12.5 \pm 0.8^{\mathbf{4}}$ & $10.1 \pm 0.8$ \\
\hline
\end{tabular}

$\Delta P<0.05$, compared with $\mathrm{T}_{0}$ in the same group; $\# p<0.05$, compared with the control group 
Table 4: Cortisol and IL-6 levels of the patients at each time point (mean \pm SD)

\begin{tabular}{lccccc}
\hline \multirow{2}{*}{ Group } & Index & $\mathbf{T}_{\mathbf{0}}$ & $\mathbf{T}_{\mathbf{1}}$ & $\mathbf{T}_{\mathbf{2}}$ & $\mathbf{T}_{\mathbf{3}}$ \\
\hline \multirow{2}{*}{ Study } & Cortisol $(\mu \mathrm{g} / \mathrm{mL})$ & $13.1 \pm 3.9$ & $17.2 \pm 4.1 \#^{\mathbf{\Lambda}}$ & $24.4 \pm 4.8 \#^{\mathbf{\Lambda}}$ & $19.5 \pm 3.1 \#^{\mathbf{\Lambda}}$ \\
\multirow{2}{*}{ Control } & IL-6 $(\mathrm{pg} / \mathrm{mL})$ & $15.3 \pm 2.8$ & $26.5 \pm 4.8 \#^{\mathbf{\Lambda}}$ & $30.4 \pm 5.0 \#^{\mathbf{\Lambda}}$ & $24.0 \pm 5.9 \#^{\mathbf{\Lambda}}$ \\
& Cortisol $(\mu \mathrm{g} / \mathrm{mL})$ & $12.5 \pm 4.0$ & $24.4 \pm 5.5^{\mathbf{\Lambda}}$ & $38.2 \pm 6.5^{\mathbf{\Lambda}}$ & $23.4 \pm 4.8^{\mathbf{\Lambda}}$ \\
& IL-6 $(\mathrm{pg} / \mathrm{mL})$ & $15.0 \pm 2.9$ & $33.2 \pm 5.7^{\mathbf{\Lambda}}$ & $42.5 \pm 6.8^{\mathbf{\Delta}}$ & $35.1 \pm 6.8^{\mathbf{\Lambda}}$ \\
\hline
\end{tabular}

$\Delta P<0.05$, compared with $\mathrm{T}_{0}$ in the same group; $\# p<0.05$, compared with the control group

Table 5: T-cell subsets in the two groups at various time points (mean \pm SD)

\begin{tabular}{lccccc}
\hline Group & Index & $\mathbf{T}_{\mathbf{0}}$ & $\mathbf{T}_{\mathbf{1}}$ & $\mathbf{T}_{\mathbf{2}}$ & $\mathbf{T}_{\mathbf{3}}$ \\
\hline \multirow{3}{*}{ Study } & CD3+ (\%) & $60.1 \pm 6.3$ & $59.2 \pm 6.1$ & $58.1 \pm 7.2 \#$ & $54.5 \pm 8.2 \#^{\mathbf{\Lambda}}$ \\
& CD4+ (\%) & $41.3 \pm 4.8$ & $40.5 \pm 4.1$ & $37.9 \pm 6.4 \#^{\mathbf{\Lambda}}$ & $36.0 \pm 7.9 \#^{\mathbf{\Lambda}}$ \\
& CD8+ (\%) & $20.5 \pm 7.8$ & $21.0 \pm 5.9$ & $20.1 \pm 7.1$ & $19.2 \pm 8.3$ \\
& CD4+/CD8+ & $2.11 \pm 0.11$ & $2.08 \pm 0.12$ & $2.05 \pm 0.14 \#^{\mathbf{\Lambda}}$ & $1.90 \pm 0.13 \#^{\mathbf{\Lambda}}$ \\
Control & CD3+ (\%) & $60.4 \pm 6.5$ & $58.9 \pm 7.2$ & $50.3 \pm 8.3^{\mathbf{\Lambda}}$ & $40.5 \pm 9.1^{\mathbf{\Lambda}}$ \\
& CD4+ (\%) & $41.5 \pm 5.2$ & $39.9 \pm 5.4$ & $28.1 \pm 6.5^{\mathbf{\Lambda}}$ & $21.1 \pm 8.6^{\mathbf{4}}$ \\
& CD8+(\%) & $20.2 \pm 7.4$ & $20.5 \pm 6.3$ & $22.4 \pm 8.0$ & $19.4 \pm 9.3$ \\
\hline
\end{tabular}

$\Delta P<0.05$, compared with $\mathrm{T}_{0}$ in the same group; $\# p<0.05$, compared with the control group

Comparison of incidence of postoperative agitations between the two groups

There were 2 children ( $8 \%$ ) with agitation in the study group, and $9(36 \%)$ in the control group ( $p$ $=0.037$ )

\section{DISCUSSION}

Congenital anal atresia is a malformation due to abnormal intestine migration at the late stage of fetal growth. More than half of the affected children have fistulas in the rectum and genitourinary organs where feces can go through, so they have to receive surgical treatment as soon as they are born [7]. However, the surgery easily gives rise to perioperative pain and stress responses due to multiple-organ involvement, long operation time, and long incision. Newborns have a hypoplastic and regular system of pain, and react very strongly to pain. Their nociception neuroendocrine - cardiovascular central regulator of pain stress is 3 to 5 times stronger than that of adults. This amplifies their reaction to pain in the postoperative life, by accentuating their immune response [8].

At the same time, pain stimulation in newborns makes them assume postures to alleviate the pain. If these postures are not corrected, they will negatively impact their limbs and growth. Consequently, it is necessary that the anesthesia used maintains blood pressure and heart rate, removes significant stress reflexes during surgery, enhances awakening of the patient, and prevents nausea and vomiting. In addition, the anesthesia should not influence postoperative healing of the inevitable surgical incision mark. Currently, general anesthesia for the newborn is usually carried out with combination of sevoflurane and remifentanil. Sevoflurane is widely used as surgery anesthesia for adults and children because of its rapid effect and low inhibition on stress response. However, one of its common adverse reactions is postoperative agitation [9]. Remifentanil has a strong analgesic effect, but it is associated with high incidence of postoperative hyperalgia [10]. Hence, it is necessary to develop a more suitable anesthesia for use in surgery for congenital anal atresia, so as to achieve better inhibition of perioperative stress response.

Dexmetidine hydrochloride (DXM), a new narcotic analgesic, is an adrenal receptor agonist with high selectivity. The drug which has good analgesic and sedative effects, stimulates $\alpha 2$ receptors in the synaptic upper segment of the brainstem locus coeruleus in the central nervous system, resulting in the release of noradrenaline. In the p-resent study, the arterial pressure and heart rate of the control group at the beginning and end of surgery were significantly increased, but there were no significant differences in these indexes at various time points in the observation group. Moreover, the arterial pressure and heart rate of the observation groups were much lower at the beginning and end of surgery. Thus, DXM depresses stress response and stabilizes intraoperative hemodynamics by reducing the stimulation of sympathetic nerves. This was also reflected in the intraoperative blood loss of the two groups. Stable blood pressure and heart rate decrease blood loss during surgery. Thus, the observation group has less blood loss than the 
control group, which is of advantage in the children's recovery.

Cortisol and IL-6 levels in both groups were elevated at $T_{1}, T_{2}$ and $T_{3}$, relative to their levels at $T_{1}$, and were significantly lower in the observation group than in the control group. Cortisol is one markers of stress response and its level reflects the severity of stress response. The body releases IL-6, an inflammatory factor, in response to tissue injury. During surgery, IL-6 regulates immune response and activates inflammatory cascade. Thus, it can be regarded as a marker of the magnitude of stress response in newborns. The findings of the current study reveal that DXM was able to reduce the stress response and inflammatory reaction due to surgery-induced pain, which is in accordance with previous reports [11]. In both groups, CD3 +, CD4 + and CD4 + / CD8 + at $\mathrm{T}_{2}$ and $\mathrm{T}_{3}$ were increased, showing that surgery can impair immune balance in the children, resulting in immunosuppression and imbalance in T-cell subsets. Some studies have reported that surgery-induced stress response may lead to immuno-dissonance and immuno-suppression, and that the degree of suppression is associated with the magnitude of surgical stress [12]. Relative to the control group, the observation group had much higher levels of CD3 +, CD4 + and CD4 + / CD8+ at $\mathrm{T}_{2}$ and $\mathrm{T}_{3}$. This suggests that the observation group had less immunodissonance and immuno-suppression than the control group. Thus, DXM can enhance the recovery of immune function, which is related to its reduction of stress response. Two cases of postoperative agitation were seen in the observation group, which were significantly lower than 9 similar cases in the control group. However, there was no significant difference in time taken for recovery from anesthesia between the two groups. It can be reasonably suggested that DXM can lessen postoperative agitation in children, through its sedative and analgesic effects due to inhibition of nervous system excitability. The effect of DXM may also be related to its ability to restrain remifentanilinduced hyperalgesia, indirectly indicating its role in decreasing surgical stress response. In addition, DXM does not over-inhibit nerves, so it has no effect on the normal recovery of consciousness.

\section{CONCLUSION}

The combination of traditional anesthesia with dexmedetomidine hydrochloride is more suitable for neonatal anal atresia than traditional routine anesthesia alone. Due to its good anesthetic effect and stable hemodynamics, the combination achieves better control of stress response-induced inflammatory reactions and immunosuppression. In addition, it has fewer adverse reactions than traditional anesthesia.

\section{DECLARATIONS}

\section{Conflict of Interest}

No conflict of interest associated with this work.

\section{Contribution of Authors}

We declare that this work was done by the author(s) named in this article and all liabilities pertaining to claims relating to the content of this article will be borne by the authors, all authors read and approved the manuscript for publication. Fang Yin conceived and designed the study, Aihua Liu, Lichuan Tian collected and analysed the data, Aihua Liu wrote the manuscript.

\section{REFERENCES}

1. Gao $X Y$, Gao PM, Wu SG, Mai ZG, Zhou J, Huang $R Z$, Zhang ST, Zhong HQ, Liao YM, Zhang AM, et al. Risk factors for congenital anal atresia. Chin J Contemp Pediatr 2016; 18 (6): 541-544.

2. Wang W, Liu G, Yu MN. Two-stage treatment for high/intermediate-type anorectal malformation through laparoscopy anorectoplasty. Chin J Pediatr Surg 2015; 36 (6): 405-408.

3. Zhang JS, Li L, Diao M. Transumbilical Single-incision Laparoscopic Assisted Anorectoplasty for Middle and High Imperforate Anus. Chin J Minim Inva Surg 2016; 16 (5): 424-427.

4. Chen LJ, Xue QS, Yu BW. Neuroprotection and mechanisms of dexmedetomidine. Chin Pharmacol Bulletin 2015; 11: 1493-1496.

5. Pasin L, Febres D, Testa V, Frati E, Borghi G, Landoni G, Zangrillo $A$. Dexmedetomidine vs midazolam as preanesthetic medication in children: a meta-analysis of randomized controlled trials. Paediatric Anaesthesia 2015; 25 (5): 468-476.

6. World Health Organization. Declaration of Helsinki. $\mathrm{Br}$ Med J 1996; 313(7070): 1448-1449.

7. Zhuo B, Zhang $H$, Yin Y, Ma T, Liu F, Cao H, Zou $H$. Rectal mucosa stripping and pull-through from rectal muscle sheath of blind pouch in the treatment of congenital high anal atresia in the newborn. Chin $J$ Gastrointest Surg 2014; 17 (1): 81-84.

8. Mao CM, Ge L, He XQ. Efficacy of Caudal Block with Dexmedetomidine and Ropivacaine for Postoperative Analgesia in pediatric. Jinagsu Med J 2015; 41 (9): 1096-1097.

9. Wen $X J$, Liang $H$, Yang $C X$. Comparison of anesthetic efficacy of ketamine versus sevoflurane for foreskin 
ligation in pediatric patients. Chin J Anesthesiol 2016; 36 (3): 318-320.

10. Li XQ, Wang JJ, Wang Q. Meta-analysis on the effect of $N$-methyl-D-aspartate receptor antagonist on remifentanil induced hyperalgesia. J Clin Experimental Med 2017; 16 (9): 866-872.

11. Jin D, Bai $Y, W u H$. Effects of dexmedetomidine used to supplement analgesia with sufentanil on stress response and inflammatory response after cardiac valve replacement with CPB. Chin J Anesthesiol 2016; 36 (1): 49-52.

12. Xiang $M, X u G Q, M o H Z$. Effect of various anesthesia methods on perioperative immunity of the patient with cervical cancer underwent radical surgery. J Pra Med 2015; (11): 1889-1890. 